\title{
Revisão da Metodologia INTERA e sua Aplicação no Desenvolvimento de um Jogo Educacional do tipo RPG
}

\author{
Luís Felipe de O. Melle ${ }^{1}$, Juliana C. Braga ${ }^{1}$, Edson P. Pimentel ${ }^{1}$, Sílvia C. Dotta ${ }^{1}$ \\ ${ }^{1}$ Centro de Matemática Computação e Cognição (CMCC) - Universidade Federal \\ do ABC (UFABC)
}

Av. dos Estados, 5001 - Bairro Santa Terezinha - Santo André - SP - Brazil

\{luis.melle, juliana.braga, edson.pimentel, silvia.dotta\}@ufabc.edu.br

\begin{abstract}
The development of educational games requires a multidisciplinary team's involvement to balance pedagogical aspects, gameplay, usability, and accessibility. Several methodologies designed for game development have been applied with educational games, but they have gaps. These gaps also occurred with the INTERA methodology, when used in the development of a role-playing game (RPG). This work aims to present a review of the INTERA methodology, including elements to guide and organize the development of educational digital games. Additionally, the article also presents a field study of applying the new INTERA version in an RPG-type game.
\end{abstract}

\begin{abstract}
Resumo. O desenvolvimento de jogos educacionais necessita do envolvimento de uma equipe multidisciplinar de modo a equilibrar aspectos pedagógicos, de jogabilidade, usabilidade e também de acessibilidade. Diversas metodologias concebidas para o desenvolvimento de jogos têm sido aplicadas com jogos educacionais mas apresentam lacunas. Isto também ocorreu com a metodologia INTERA, quando utilizada no desenvolvimento de um jogo do tipo Role-Play Game (RPG). Este trabalho tem por objetivo apresentar a revisão da metodologia INTERA incluindo elementos para guiar e organizar o desenvolvimento de jogos digitais educacionais. Adicionalmente, o artigo apresenta também um estudo de campo de aplicação da nova versão INTERA em um jogo do tipo RPG.
\end{abstract}

\section{Introdução}

Jogos Educacionais são aqueles que quando projetados já são pensados como serão aplicados e quais seus propósitos educacionais, no qual os usuários poderão compreender conceitos, desenvolver habilidades e resolver problemas. Os jogos educacionais podem ser aplicados de forma informal e com ou sem a supervisão de alguém [Cojocariu e Boghian 2014].

O desenvolvimento de um jogo educacional exige participação de equipe multidisciplinar e a integração de diversos elementos como: pedagógicos, imagens, softwares e código-fonte. Para garantir a qualidade de um jogo é necessária a adoção de métodos, normas e padrões profissionais [Lavor 2009]. Jogos Educacionais devem possuir um bom projeto para alcançar seus propósitos principais, pois um jogo sem critérios bem definidos pode acabar apresentando resultados ilusórios. Considerando a importância de uma boa metodologia para o desenvolvimento dos jogos digitais educacionais e a relevância destes 
para a educação e seus benefícios no processo do ensino-aprendizagem, será proposto a adaptação de uma metodologia para o desenvolvimento desses jogos.

Existem metodologias com foco no desenvolvimento de jogos educacionais. Algumas são específicas para determinados conteúdos como herança cultural [Andreoli et al. 2017], ensino de programação [Malliarakis et al. 2014] ou voltadas apenas para um tipo de jogo pré-definido, como os jogos de simulação [Rocha et al. 2014]. Também não consideram diferentes tipos de usuários [Cano et al. 2016], como, por exemplo, pessoas com deficiência. Já outras metodologias focam somente no design de jogos, mas negligenciam etapas da engenharia de software e/ou aspectos educacionais. Além disso, as metodologias específicas para jogos educacionais, carecem de documentação, e portanto, são difíceis de serem utilizadas. A metodologia INTERA foi desenvolvida com o propósito de atender requisitos pedagógicos e de sistemas e com foco na documentação por meio dos artefatos produzidos no decorrer do processo.

A INTERA foi escolhida neste trabalho pelos seguintes motivos: contém documentação, como livros e cursos, contempla todas as etapas de engenharia de software [Melle et al. 2019], contém aspectos pedagógicos bem definidos [Braga et al. 2013] e tem sido bem aceita na comunidade de informática na educação. A metodologia INTERA já foi aplicada em contexto de jogos educacionais, inclusive de jogos do tipo RolePlay Game (RPG) [Nishida et al. 2014]. No entanto, algumas lacunas foram identificadas tais como: ausência de alguns papéis específicos para desenvolvimento de jogos, ausência das práticas e artefatos gerados no desenvolvimento de um jogo educacional.

Este trabalho tem por objetivo apresentar a revisão da metodologia INTERA incluindo elementos para guiar e organizar o desenvolvimento de jogos digitais educacionais. Adicionalmente, o artigo apresenta também um estudo de campo de aplicação da nova versão INTERA em um jogo do tipo RPG. O artigo está organizado da seguinte forma: a seção 2 posiciona os conceitos fundamentais sobre os elementos dos jogos e a seção 3 discute alguns trabalhos relacionados; a seção 4 descreve a metodologia e a seção 5 discute os resultados; a seção 5 estabelece as considerações finais.

\section{Fundamentos Conceituais}

Os principais conceitos necessários para a compreensão deste trabalho estão relacionados a Tétrade elementar dos jogos, que é uma teoria que caracteriza o jogo digital em 4 elementos básicos descritos a seguir: mecânica, narrativa, estética e tecnologia [Schell 2008].

A mecânica está relacionada às regras e procedimentos do jogo. As regras estão ligadas ao objetivo do jogador dentro do jogo, isto é, o que é preciso fazer para alcançá-lo e o que acontece quando falha. Também são descritas as ações que o jogador não pode fazer. A narrativa está ligada diretamente com as mecânicas e estética do jogo, que juntas servem para fortalecer a história, dando-lhe mais vida. A estética está relacionada aos elementos visuais e sonoros jogo, sendo um elemento de contato mais direto com o jogador. Para que a estética seja apresentada de forma adequada ela precisa estar inteiramente ligada as histórias do jogo. A tecnologia do jogo não está ligada diretamente a um computador ou algo do gênero, e sim a materiais e interações que faça o jogo existir, podendo ser desde um lápis e papel até um laser. A tecnologia precisa ser bem analisada e escolhida, pois após sua escolha será possível fazer algumas e limitar outras. A partir da 
tecnologia a história do jogo será contada, seguindo a estética e mecânica desenvolvidas.

Apesar do jogo ser um software, os jogos são mais complexos que isso, já que para o seu desenvolvimento é exigido inspiração artística determinada principalmente pelos elementos de história e estética. A inspiração artística pode ser considerada um dos pontos responsáveis pelo sucesso e pela qualidade do jogo. Então, além das questões de engenharia de software, é necessário analisar o game design ${ }^{1}$ do jogo também [Morais 2009].

É importante esclarecer as diferenças entre jogos, jogos educacionais e softwares, para compreender suas peculiaridades durante seus processos de desenvolvimento. Para tal, o Quadro 1 foi elaborado baseado na tétrade elementar descrita na seção 2, e na definição clássica de software. Esse Quadro, denota com um “x” os elementos considerados primordiais para caracterizar a diferença entre cada um deles.

Quadro 1. Diferenças entre Software, Jogos e Jogos Educacionais.

\begin{tabular}{|l|c|c|c|}
\hline Características & Software Comum & Jogos & Jogos Educacionais \\
\hline Possui Código-Fonte & $\mathrm{x}$ & $\mathrm{x}$ & $\mathrm{x}$ \\
\hline Executa tarefas automaticamente & $\mathrm{x}$ & $\mathrm{x}$ & $\mathrm{x}$ \\
\hline Resolve problemas automaticamente & $\mathrm{x}$ & $\mathrm{x}$ & $\mathrm{x}$ \\
\hline Tem Mecânica & - & $\mathrm{x}$ & $\mathrm{x}$ \\
\hline Tem Estética & + & $\mathrm{x}$ & $\mathrm{x}$ \\
\hline Tem Tecnologia & $\mathrm{x}$ & $\mathrm{x}$ & $\mathrm{x}$ \\
\hline Tem Narrativa & - & $\mathrm{x}$ & $\mathrm{x}$ \\
\hline Possui Elementos pedagógicos & - & - & $\mathrm{x}$ \\
\hline
\end{tabular}

Observa-se, pelo Quadro 1, que a narrativa e a mecânica não precisam existir dentro de um software comum e a estética existe, mas não é primordial. Já os elementos pedagógicos aparecem apenas nos jogos educacionais, e nesse caso, o componente pedagógico torna o desafio em alcançar a qualidade do jogo ainda maior, uma vez que a qualidade desse produto pode afetar o processo de aprendizagem que aquele jogo pretende mediar. Não obstante, para jogos educacionais, é mandatório adoção de uma metodologia que possa sistematizar seu desenvolvimento em busca de maior qualidade.

\section{Trabalhos Relacionados}

Nessa seção são discutidos 13 trabalhos relacionados a esse artigo. Foram considerados trabalhos relacionados aqueles que propõem framework, metodologia, processo ou design para o desenvolvimento de jogos sérios. Os trabalhos apresentados foram selecionados a partir de revisão sistemática de literatura e pesquisas no Google Acadêmico. Foram analisados os seguintes aspectos dos jogos educacionais: características dos jogos [Schell 2008], gestão de projetos, papel da equipe que compõe o jogo, características educacionais, participação do usuário incluindo questões de acessibilidade. Esses aspectos foram definidos a partir do estudo realizado no trabalho de [Melle et al. 2019]. Após a apresentação do quadro, inicia-se análise de acordo com as características supracitadas debatendo cada trabalho com a metodologia INTERA, escolhida para este trabalho.

\subsection{Tétrade}

A partir dos 4 elementos da tétrade apresentada na seção 2, os 13 trabalhos foram analisados. Observou-se que, mesmo que um jogo possar ser claramente dividido nos qua-

\footnotetext{
${ }^{1}$ Trata-se da prática de decidir tudo o que um jogo deve ser [Schell 2008].
} 
tro elementos, nenhum dos trabalhos citou explicitamente a utilização da tétrade. Apesar disso, com a análise mais minuciosa dos artigos, foi possível notar que um trabalho [Rocha et al. 2014] tem relação não muito evidente com os 4 elementos da tétrade. Os demais não fizeram referência com todos os elementos da tétrade, isso dificulta entender em que momento do processo do desenvolvimento do jogo é necessário iniciar a elaboração de cada um desses elementos e por quem. Todos os trabalhos fazem relação com a Tecnologia e apenas três dos trabalhos não fazem relação com a Estética.

Apesar de os trabalhos não deixarem explícito esses elementos em sua proposta, foi possível notar alguns pontos que fazem alguma relação, como: [Andreoli et al. 2017] cita a utilização do mapa de jogos e cena lógica que faz relação com a mecânica e o storytelling que tem relação a narrativa. [Saavedra et al. 2014] cita o documento de game design que pode conter elementos de mecânica, narrativa tecnologia e estética. [Cano et al. 2015], em relação à mecânica, comenta o design de jogos, níveis de dificuldade do jogo, personagens e elementos de interação. E em relação à narrativa comenta sobre a história do jogo em uma de suas etapas. [de Lope et al. 2017] comenta sobre o design de desafios nos elementos de mecânica e o design de cena e capítulo nos elementos de narrativa. [Olszewski et al. 2017] cita a criação de storyboards que descreve o fluxo e funcionalidades do jogo (mecânicas). [Battistella et al. 2016] em relação às mecânicas foi comentado a modelagem das etapas do jogo e os feedbacks para o jogador e a narrativa somente é citada em uma de suas fases. E o [Aslan e Balci 2015] apenas cita a narrativa em uma de suas fases, porém não é detalhado. A metodologia INTERA não apresenta nenhuma menção explícita à tétrade. Por outro lado, o elemento tecnologia está bastante presente, o que é muito comum para softwares educacionais, não apresentando, assim, novidades nesse sentido.

\subsection{Gestão de projetos}

Em 10 trabalhos, o planejamento acontece apenas nas etapas iniciais do projeto, Apenas no trabalho [Alcover et al. 2018] é mencionado uma gestão de projetos sendo realizada em todo o ciclo. Os trabalhos [Cano et al. 2016] não deixa se existe o planejamento e no trabalho [Battistella et al. 2016] cita o planejamento apenas na fase de avaliação pedagógica. A metodologia INTERA menciona que a gestão de projetos deve ser realizada durante todo o ciclo de vida do projeto, e é essencial dentro de um projeto de desenvolvimento, especialmente por esse processo necessitar de equipe multidisciplinar.

\subsection{Participação do jogador no projeto e desenvolvimento do jogo e acessibilidade}

Em relação a participação do jogador durante todo o processo de desenvolvimento, apenas 4 modelos citam isso. Em [Araujo et al. 2017] é mencionado que os estudantes produziam os jogos, logo eles participam de todo o processo. Em outro, o jogador participa desde a contextualização do projeto até as etapas finais. Em relação a metodologia INTERA, não fica explícito a participação do usuário durante o processo. Nenhum trabalho menciona questões de acessibilidade.

\subsection{Papéis}

Entende-se por papeis, as funções que um ou mais membro da equipe podem desempenhar. Essa clareza é importante existir de antemão para um bom planejamento de custos e perfil de contratação das equipes. Apesar da importância dos papéis na metodologia, um 
trabalho ([Malliarakis et al. 2014]) não deixa claro quais papéis são utilizados na metodologia. Nos outros trabalhos são citados os papéis, porém são citados de forma dispersa e de difícil compreensão. Há também diferentes funções em cada modelo e observa-se que há incompletude nesse aspecto. Na metodologia INTERA, fica explícito quais papeis membros da equipe precisam ter e sua atuação no projeto. Por outro lado, a metodologia INTERA não cita alguns papeis importantes relacionados ao desenvolvimento de jogos.

\subsection{Aspectos educacionais}

Para analisar os aspectos pedagógicos de cada trabalho, foi elaborado um quadro de acordo com o trabalho [Braga et al. 2012] com atividades educacionais que um projeto de jogo educacional deveria conter, e assinalado quais dos 13 trabalhos possuíam essas atividades. Apenas um trabalho ([Barbosa Neto 2012]) contempla todas as atividades educacionais. esse trabalho que contempla todas as atividades tem como base o modelo ADDIE que se trata de uma metodologia específica para o design instrucional. Nos trabalhos de [Malliarakis et al. 2014], [Olszewski et al. 2017] e [Barbosa Neto 2012] não fica claro se possuem alguma atividade de design instrucional, ou seja, nenhuma das atividades educacionais analisadas foram encontradas nesses trabalhos. A metodologia INTERA, por se basear no ADDIE, possibilita que todas as atividades educacionais possam ser executadas no projeto, porém não considera isso uma obrigatoriedade.

\subsection{Documentação}

Um ponto essencial que está presente apenas na metodologia INTERA é a documentação detalhada. Com a documentação da metodologia, fica possível um leitor replicar a utilização em seu projeto.

\section{Metodologia}

A metodologia do trabalho dividiu-se em 3 etapas descritas como segue:

Na etapa 1, foi realizada revisão de literatura em busca de metodologias, processos ou framewoks para o desenvolvimento de jogos educacionais. A partir dos artigos encontrados, foram elaborados quadros comparativos para verificar as características, vantagens e desvantagens entre eles e também em relação a metodologia INTERA. Parte da metodologia foi publicada em [Melle et al. 2019] e parte do resultado pode ser visto na seção 3 deste trabalho. Como síntese dessa etapa, gerou-se o Quadro 2, que apresenta na coluna 1, as características que foram encontradas nos 13 trabalhos relacionados. Todas essas características foram discutidas, sendo que as principais delas foram apresentadas e debatidas na seções 2 e 3. O Quadro 2, também sinaliza se a característica é ou não suficiente na INTERA. Aquelas que não forem, serão incorporadas nesse trabalho.

A etapa 2 consistiu em acrescentar à metodologia INTERA aquelas características encontradas em outras metodologias, porém não contempladas na INTERA, são elas: Narrativa, Mecânica, Estética, Participação do Jogador e Papeis específicos de jogos. Estes pontos foram adicionados, pois são importantes no desenvolvimento de jogos digitais educacionais e à INTERA não os contemplava.

$\mathrm{Na}$ etapa 3, foi realizado um estudo de campo de caráter qualitativo e de natureza exploratória, para verificar a viabilidade de aplicação da metodologia INTERA, adaptada com as características acima, no desenvolvimento de um jogo educacional do tipo RPG. 
IX Congresso Brasileiro de Informática na Educação (CBIE 2020)

Anais do XXXI Simpósio Brasileiro de Informática na Educação (SBIE 2020)

Quadro 2. Características apresentadas ou não na metodologia INTERA.

\begin{tabular}{|l|c|}
\hline Característica & INTERA \\
\hline Narrativa & Não \\
\hline Mecânica & Não \\
\hline Estética & Não \\
\hline Bases Teóricas & Sim \\
\hline Testes & Sim \\
\hline Gestão de Projetos & Sim \\
\hline Participação do Jogador & Não \\
\hline Aspectos Pedagógicos & Sim \\
\hline Papeis específicos de jogos & Não \\
\hline Aspectos Educacionais & Sim \\
\hline Papel do Docente & Sim \\
\hline Reúso & Sim \\
\hline
\end{tabular}

O objetivo desse estudo, foi verificar se a ampliação realizada na metodologia INTERA seria suficiente para o desenvolvimento de jogos educacionais do tipo RPG, e, caso não fosse, calibrar o necessário.

Esse estudo teve duração de 18 meses e teve como objeto de estudo o jogo "Expedição Antártica" (Figura 1), um jogo educacional para o ensino interdisciplinar de ciências, matemática e educação científica.

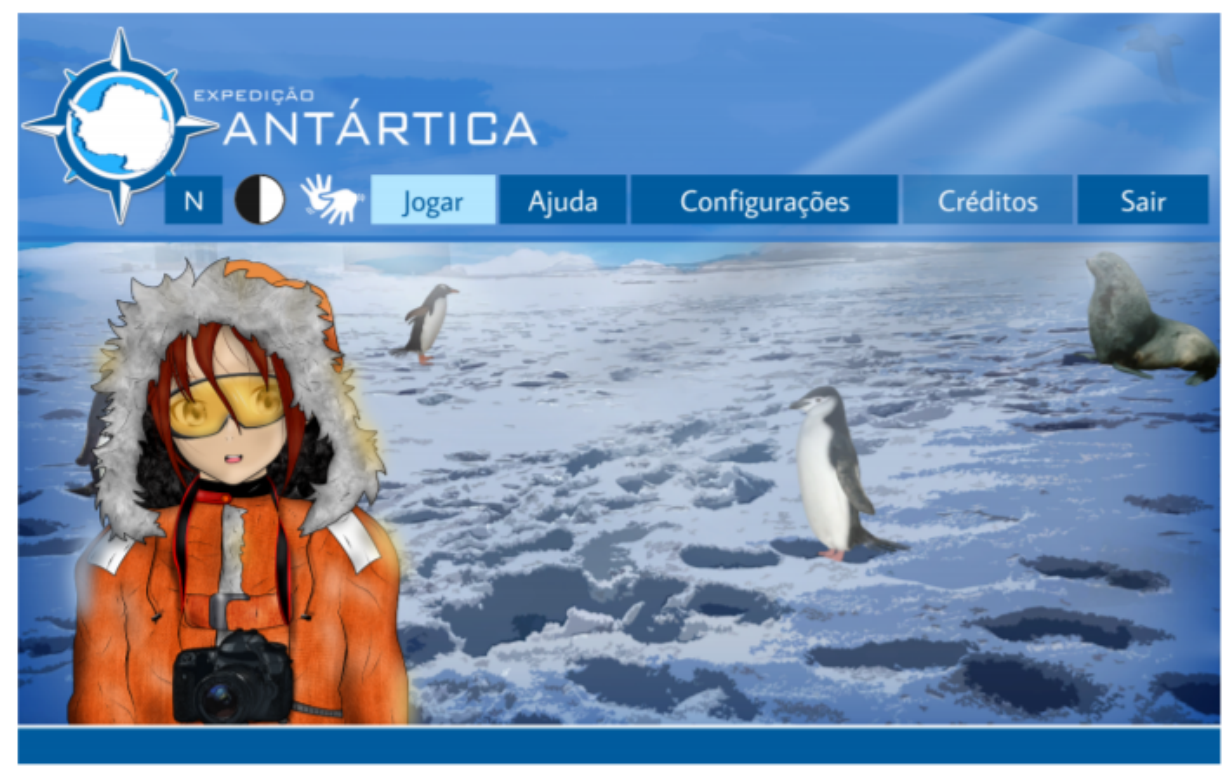

Figura 1. Tela de abertura do jogo "Expedição Antártica".

O estudo foi aplicado em um projeto para o desenvolvimento de um jogo educacional financiamento pela CAPES (Coordenação de Aperfeiçoamento de Pessoal de Nível). O jogo é do gênero RPG, no qual o jogador assumirá o papel de um turista que irá colaborar em processos de pesquisas científicas na Antártica. Dentro do jogo, existem Mini Jogos (MJ) com problemas interdisciplinares e desafios que demonstram o processo de pesquisa científica por meio de Ciência Cidadã (CC). 
Em torno de 40 pessoas participaram do desenvolvimento do jogo. Essas pessoas não necessariamente estavam envolvidas em todo o tempo de projeto. Ressalta-se que como o jogo foi desenvolvido sob a perspectiva do design universal, fizeram parte da equipe pessoas com deficiência visual e cognitiva e surdas. Esse fator foi importante para avaliar tanto a participação dos usuários durante a aplicação da metodologia INTERA revisitada como a acessibilidade seria tratada dentro desse processo. Os autores deste artigo atuaram como conteudistas, analistas, coordenadores e observadores do processo.

O trabalho utilizou fontes de dados geradas ao longo do processo de desenvolvimento do jogo. Algumas delas foram usadas para identificar em qual etapa do desenvolvimento de jogos elas deveriam ser geradas, dentre elas citam-se as principais: vídeos em LIBRAS, código-fonte do jogo, documentos de audiodescrição, especificação de requisitos, de caso de uso, documentos sobre o design de jogos, documentos sobre modelagem de sistemas computacionais $\mathrm{UML}^{2}$, diagrama de entidade relacionamento. Outras fontes foram usadas para criação de modelos de artefatos da metodologia INTERA e que serão disponibilizados no site do projeto para serem reutilizadas. Outro tipo de fonte de dados usada foi o documento de Post-Mortem do jogo, que foi gerado a partir de conversas e aplicação de um questionário com os membros das equipes, para entender as atividades realizadas por cada um deles, artefatos gerados em cada equipe, técnicas e ferramentas e sugestões de melhorias. No total foram geradas aproximadamente 45 fontes de dados.

O projeto foi aprovado no comitê de ética contendo o seguinte número de parecer: 3.537.793.

\section{Resultados}

A partir da revisão e discussão bibliográfica e posterior estudo de campo, chegou-se a uma extensão da metodologia INTERA para jogos educacionais do tipo RPG. Essa metodologia será apresentada nessa sessão em 3 tópicos: fases, papeis, etapas e artefatos.

\subsection{Fases}

As fases são períodos que organizam as etapas da metodologia INTERA em Inicial, Intermediária ou de Transição. As fases são sequenciais e definidas pela entrega de algum componente do jogo. Na maioria dos trabalhos encontrados, os jogos educacionais também possuem também 3 fases que correspondem respectivamente as fases da metodologia INTERA, porém como nomes distintos, sendo os mais usados: pré-produção, produção e pós-produção. Devido a essa correspondência, não houve necessidade de adaptar as fases da metodologia INTERA para jogos permanecendo assim as 3 fases originais.

\subsection{Papéis}

Os papeis descrevem como as pessoas devem atuar no projeto e quais são suas funções, sendo que uma pessoa pode assumir mais de um papel na metodologia. Os papeis que foram adicionados a partir desse estudo foram: Ilustrador, Designer de som, Usuários, Designer de jogos/ game designer, equipe de vídeo, equipe de acessibilidade, como audiodescritor e tradutor de libras. Os demais papeis da metodologia descritos em [Braga et al. 2013] e [Braga 2015] foram mantidos. A descrição detalhada de cada fase pode ser visualizada na página da metodologia.

\footnotetext{
${ }^{2}$ Unified Modeling Language.
} 
IX Congresso Brasileiro de Informática na Educação (CBIE 2020)

Anais do XXXI Simpósio Brasileiro de Informática na Educação (SBIE 2020)

\subsection{Artefatos}

Em quase todas as etapas da metodologia, dados são gerados pela equipe envolvida, desde documentos, planilhas, imagens até o código-fonte. Todos esses dados contendo informações são considerados artefatos para a metodologia INTERA. Foram muitos os artefatos adicionados e por limitações de espaço, serão citados os principais: documento de game design, vídeos em LIBRAS, texto de audiodescrição, arquivos de sons, caso de uso acessível, glossário em LIBRAS, diálogos dos personagens. Com o estudo de campo ficou claro que alguns artefatos passam pelas mesmas etapas da metodologia. Os artefatos estão organizados no site da metodologia.

\subsection{Etapas}

São coleções de atividades relacionadas a uma área de interesse principal. As etapas podem ocorrer concomitantemente ou não, e suas durações variam de acordo a complexidade e tipo do jogo. As etapas da metodologia INTERA são: contextualização, requisitos, arquitetura, desenvolvimento, testes e qualidade, disponibilização, gestão de projetos, ambientes de padrões e avaliação pedagógica. Após o estudo de campo, análise dos trabalhos relacionados e reuniões com o grupo de pesquisa, ficou claro que as etapas que a metodologia INTERA possui já são suficientes para o desenvolvimento de um jogo digital do tipo RPG. No entanto, foi necessário detalhar como a tétrade e a acessibilidade ocorrem em cada etapa e quais os artefatos de jogos educacionais gerados em cada uma delas.

A Figura 2 mostra a intensidade das etapas no desenvolvimento de cada elemento da tétrade e acessibilidade, no qual são os principais elementos inseridos na metodologia INTERA. Do lado esquerdo da figura estão presentes os elementos da tétrade e acessibilidade, na parte superior estão as etapas. A intensidade é presentada pelas faixas coloridas que podem oscilar de 0 à 100. Pelo gráfico, consegue-se observar as característica incremental da INTERA, pois nenhuma das etapas deixa de ser executada durante alguma fase. Também é possível notar que nas etapas de Teste e Qualidade, Disponibilização, Avaliação e Gestão de projetos atingem a intensidade $100 \mathrm{em}$ todos elemento, pois todos têm peso igual, isto é, nenhum é mais importante que outro nessas etapas.

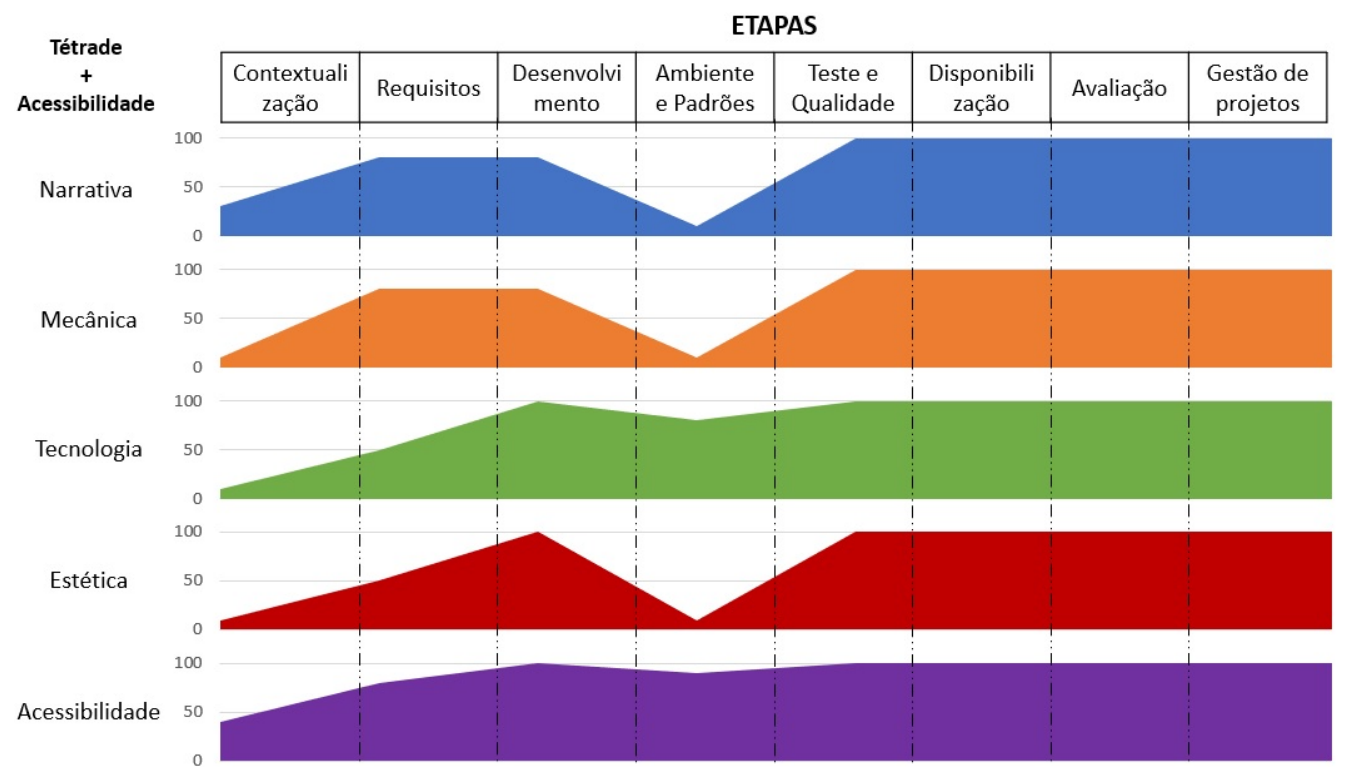

Figura 2. Etapas da metodologia em relação aos elementos da tétrade. 
IX Congresso Brasileiro de Informática na Educação (CBIE 2020)

Anais do XXXI Simpósio Brasileiro de Informática na Educação (SBIE 2020)

\section{Considerações Finais}

Este trabalho teve como objetivo estender a metodologia INTERA para o desenvolvimento de jogos educacionais do tipo RPG. O artigo discute que existe uma carência de metodologias de produção de jogos educacionais que levem em consideração ao mesmo tempo as características de software, de jogos e de objetos de aprendizagem e que sejam específicas para RPG.

Como método, partiu-se da metodologia INTERA, já existente, que sofreu alterações baseado em estudo bibliográfico e também em um estudo de campo. Após a finalização do estudo de campo, foram acrescentados elementos de acessibilidade a metodologia, todos eles voltados ao Design Universal. Esses elementos não foram encontrados na literatura e por isso só foram incorporados após estudo de campo. Alguns desses elementos foram: papel do audiodescritor, tradutor de libras, artefato de caso de uso acessível, artefatos de libras e audiodescrição. Outros aspectos incorporados na metodologia INTERA foram: os 4 elementos da tétrade, participação dos usuários durante todo o processo e papéis específicos para jogos. Essa nova versão da INTERA pretende propiciar que jogos educacionais do tipo RPG possam ser desenvolvidos usando um "passo a passo" que levem a produção de jogos educacionais que contribuam de forma efetiva para a aprendizagem, mas não deixem de lado o engajamento que o jogo proporciona. A metodologia possui como diferencial uma documentação que possibilita seu entendimento e sua aplicação disponível em: www.interaufabc.com.br/intera-rpg. Como trabalhos futuros, pretende-se verificar se a metodologia especificada e validada em um jogo do tipo RPG, pode ser aplicada para outros tipos de jogos educacionais. Além disso, os autores estão verificando se a aprendizagem está sendo alcançada pelo jogo desenvolvido a partir dessa metodologia.

\section{Referências}

Alcover, E. A., Jaume-i Capó, A., e Moyà-Alcover, B. (2018). Progame: A process framework for serious game development for motor rehabilitation therapy. PLOS ONE, 13(5):1-18.

Andreoli, R., Corolla, A., Faggiano, A., Malandrino, D., Pirozzi, D., Ranaldi, M., e Santangelo, Gianluca e Scarano, V. (2017). A framework to design, develop, and evaluate immersive and collaborative serious games in cultural heritage. J. Comput. Cult. Herit., 11(1):4:1-4:22.

Araujo, L. F., Silva, C. d., Silva, A. P., e Machado, T. L. (2017). Metodologia de desenvolvimento de jogos digitais para o ensino técnico de nível médio.

Aslan, S. e Balci, O. (2015). Gamed: digital educational game development methodology. Simulation, 91(4):307-319.

Barbosa Neto, J. F. (2012). Uma metodologia de desenvolvimento de jogos educativos em dispositivos móveis para ambientes virtuais de aprendizagem. Master's thesis, Universidade Federal de Pernambuco.

Battistella, P. E. et al. (2016). Engaged: Um processo de desenvolvimento de jogos para ensino em computação.

Braga, J. (2015). Objetos de Aprendizagem Volume 2: metodologia de desenvolvimento. 
IX Congresso Brasileiro de Informática na Educação (CBIE 2020)

Anais do XXXI Simpósio Brasileiro de Informática na Educação (SBIE 2020)

Braga, J. C., Dotta, S., Pimentel, E., e Stransky, B. (2012). Desafios para o desenvolvimento de objetos de aprendizagem reutilizáveis e de qualidade. In Anais do Workshop de Desafios da Computação Aplicada à Educação, pages 90-99.

Braga, J. C., Pimentel, E., e Dotta, S. (2013). Metodologia intera para o desenvolvimento de objetos de aprendizagem. In Brazilian Symposium on Computers in Education (Simpósio Brasileiro de Informática na Educação-SBIE), volume 24, page 306.

Cano, S., Munoz Arteaga, J., Collazos, C. A., Gonzalez, C. S., e Zapata, S. (2016). Toward a methodology for serious games design for children with auditory impairments. IEEE Latin America Transactions, 14(5):2511-2521.

Cano, S. P., González, C. S., Collazos, C. A., Arteaga, J. M., e Zapata, S. (2015). Agile software development process applied to the serious games development for children from 7 to 10 years old. International Journal of Information Technologies and Systems Approach (IJITSA), 8(2):64-79.

Cojocariu, V.-M. e Boghian, I. (2014). Teaching the relevance of game-based learning to preschool and primary teachers. Procedia-Social and Behavioral Sciences, 142.

de Lope, R. P., Arcos, J. R. L., Medina-Medina, N., Paderewski, P., e Gutiérrez-Vela, F. (2017). Design methodology for educational games based on graphical notations: Designing urano. Entertainment Computing, 18:1-14.

Lavor, R. M. d. (2009). Metodologia utilizada no desenvolvimento de games.

Malliarakis, C., Satratzemi, M., e Xinogalos, S. (2014). Designing educational games for computer programming: A holistic framework. Electronic Journal of e-Learning, 12(3):281-298.

Melle, L. F. d. O., Braga, J. C. B., e Stiubiener, I. (2019). Estudo sobre metodologias de desenvolvimento de jogos digitais educacionais: Revisão sistemática da literatura. In Brazilian Symposium on Computers in Education (Simpósio Brasileiro de Informática na Educação-SBIE), volume 30, page 1052.

Morais, Felipe Castanheira e Silva, C. M. (2009). Desenvolvimento de jogos eletrônicos. e-xacta, 2(2).

Nishida, A. K., Braga, J. C., Monteforte, A., e Benassi, R. F. (2014). Jogo educacional sobre consumo de energia elétrica. In Brazilian Symposium on Computers in Education (Simpósio Brasileiro de Informática na Educação-SBIE), volume 25.

Olszewski, A. E., Wolbrink, T. A., e . (2017). Serious gaming in medical education: a proposed structured framework for game development. Simulation in Healthcare, 12(4):240-253.

Rocha, R. V. d. et al. (2014). Metodologia iterativa e modelos integradores para desenvolvimento de jogos sérios de treinamento e avaliação de desempenho humano.

Saavedra, A. B., Rodríguez, F. J. A., Arteaga, J. M. n., Salgado, R. S., e Ordoñez, C. A. C. (2014). A serious game development process using competency approach: Case study: Elementary school math. In Proceedings of the XV International Conference on Human Computer Interaction, Interacci\&\#243;n '14, pages 99:1-99:9, New York, NY, USA. ACM.

Schell, J. (2008). The Art of Game Design: A book of lenses. CRC press. 